\title{
CAUSAS, CONSECUENCIAS Y REGULACIÓN DEL CAPITAL GOLONDRINA: EL CASO DE COSTA RICA
}

\author{
JOSÉ FULVIO SANDOVAL VÁSQUEZ \\ Escuela Ciencias de la Administración \\ Universidad Estatal a Distancia, Costa Rica \\ jsandoval@uned.ac.cr
}

\section{RESUMEN}

El siguiente artículo analiza el ingreso de capital financiero de corto plazo (capital golondrina) en el país a partir del segundo semestre de 2012. Interesa revisar lo que establece la teoría económica sobre su origen, causas y consecuencias, así como las medidas regulatorias que pueden tomar las autoridades económicas para limitar estos flujos y contrarrestar sus efectos macroeconómicos. Finalmente, a la luz de estos desarrollos se revisa la propuesta del Poder Ejecutivo tendente a desestimular el arribo de estos capitales.

PALABRAS CLAVE: FLUJOS DE CAPITAL, TRINIDAD IMPOSIBLE, TASAS DE INTERÉS, TIPO DE CAMBIO, INFLACIÓN, RESERVAS MONETARIAS INTERNACIONALES.

\section{ABSTRACT}

In this paper we analyze the entry of shortterm financial capitals to the country in the second half of 2012. What economic theory says regarding its origin, causes and consequences is going to be reviewed, as well as the regulatory measures that policymakers can take to limit their flows and counteract their macroeconomic effects. Finally, taking into account these developments, an executive proposal aiming to discourage the arrival of these capitals is analyzed.
KEYWORDS: CAPITAL FLOWS, IMPOSSIBLE TRINITY, INTEREST RATES, EXCHANGE RATES, INFLATION, INTERNATIONAL MONETARY RESERVES.

\section{INTRODUCCIÓN}

Durante el segundo semestre de 2012 la economía costarricense comenzó a experimentar una revaloración del colón con respecto al dólar, así como una reaparición de las presiones inflacionarias, las cuales se había logrado controlar mediante la aplicación del sistema de bandas cambiarias para establecer el tipo de cambio.

El capital golondrina, capital de corto plazo o capital especulativo figura entre las principales explicaciones de este renacer inflacionario. Como ha sucedido en diversos países en desarrollo, la afluencia de estos capitales produce desequilibrios en la balanza de pagos, en el mercado cambiario y en el mercado financiero, capaces de desestabilizar cualquier economía en cualquier momento. En este sentido, para el caso de Colombia, Diago (2011) señala que los capitales golondrina han producido consecuencias negativas como la revaluación de la moneda perjudicando el esfuerzo exportador de la economía y aumentando la presión sobre los precios internos.

En el presente documento se analiza la afluencia del capital especulativo que ha tenido lugar en Costa Rica a partir del segundo semestre de 2012, sus causas, consecuencias sobre el equilibrio ma- 
croeconómico; así como las medidas propuestas para su regulación, en especial el proyecto de ley que se discute desde mediados de 2013 en la Asamblea Legislativa denominado: Ley para desincentivar el ingreso de capitales externos.

El capital golondrina o capital especulativo son flujos de capital que ingresan al país en el corto plazo atraídos por una mayor rentabilidad relativa financiera en relación con las posibilidades de inversión en el extranjero. Sin embargo, una vez que dicho capital ha realizado su ganancia o se presenta una mejor oportunidad financiera en el extranjero, sale del país hacia esos otros mercados en pos de mejores incentivos y rentabilidad (León, 2013).

El diseño de esta investigación es esencialmente de carácter descriptivo y comparativo, ya que pretende contribuir a ampliar el conocimiento del proceso de afluencia de capitales de corto plazo y sus consecuencias sobre la economía costarricense, así como analizar las medidas propuestas para contener ese fenómeno. Cabe indicar que un estudio descriptivo mide, evalúa y recolecta datos de diversos elementos, dimensiones o componentes de un fenómeno objeto de investigación (Hernández, Fernández y Batista, 2003).

El estudio es teórico utilizando fuentes bibliográficas, tanto libros y artículos de relevancia relacionados con el tema, los cuales han permitido contar con un marco de referencia para comprender el fenómeno en estudio. Asimismo, por tratarse de un tema de actualidad, parte del material proviene de publicaciones periódicas, consultadas en línea.

\section{El capital golondrina}

Como se indicó en la introducción, por capital golondrina (capital especulativo o capitales de corto plazo) se entienden aquellos fondos que provienen del mercado internacional que ingresan al sistema financiero o bursátil del país en procura de altos rendimientos o ganancias. Su permanencia en el mercado interno es por un periodo corto (máximo un año) y posterior- mente, se trasladan a otros países donde las posibilidades de ganancia sean mayores. En general, este tipo de inversiones: "vienen en las bonanzas y huyen en las crisis, de manera que agudizan los ciclos económicos. Es tal la movilidad de estos capitales especulativos, y tan grandes los montos que se pueden mover de un país a otro en fracciones de segundo, que tienen la capacidad de producir crisis financieras de enormes proporciones." (Lopera, Rojas y Sánchez, 2008, p. 2).

Para cualquier economía en desarrollo, el ingreso de capitales externos es deseable en el tanto se conviertan en inversión real, es decir, en compra de bienes de producción o edificación de infraestructura, pues contribuyen a generar empleo y riqueza en el país anfitrión. Sin embargo, los capitales de corto plazo o especulativos se invierten en el mercado financiero sin constituir formación de capital físico y su salida puede desestabilizar los mercados financieros de los países receptores de este tipo de inversiones financieras. El capital de corto plazo, generalmente, se invierte "en bonos, pagarés, certificados del gobierno y valores de empresas privadas" (Carbaugh, 2009, p. 298).

\section{Antecedentes Latinoamericanos}

Como se verá en este apartado, la experiencia Latinoamericana, en especial la de Chile, ha demostrado que ante la imposibilidad de resolver las causas internas que originan el ingreso de capitales de corto plazo, el flujo se puede controlar con una adecuada regulación, la cual se puede establecer por medio de imposiciones tributarias a las utilidades, al ingreso o a la salida de este capital, así como a través de encajes o depósitos que obligan la inmovilización de parte de esos recursos y sobre los cuales no se reconocen rendimientos.

\section{México y Argentina}

El caso de México se presentó en el año 1994 cuando su economía experimentó una fuerte devaluación al agotarse sus reservas internacio- 
nales debido a las salidas de capital financiero. Esta crisis tuvo efectos internacionales y afectó de forma negativa a todos los sectores productivos de la economía mexicana.

La crisis fue producto de una economía frágil y que presentó una salida masiva de capital golondrina que había ingresado al país atraído por sus tasas de interés y estabilidad cambiaria. Dichos capitales, por su naturaleza de corto plazo, no contribuyeron al fortalecimiento de la estructura productiva del país, sino que se utilizaron, principalmente, para financiar el consumo público y privado (Gastaldi y Ferro, 1995).

Sin embargo, los altos intereses que ofrecía el mercado financiero mexicano no eran sostenibles en el mediano plazo, pues no se estaba ampliando la base productiva y, una vez, que la situación se hizo evidente para los inversionistas, estos optaron por retirar su capital del país reduciendo al mínimo las reservas monetarias internacionales, lo que obligó a un ajuste violento vía devaluación, inflación y desempleo. Este proceso tuvo repercusiones internacionales y se le ha denominado en la literatura económica como el "Efecto Tequila" (Samuelson y Nordhaus, 2010).

Para hacer frente a esta crisis, el gobierno mexicano debió acudir a la ayuda externa, la cual se materializó en la apertura de una línea de crédito por US\$20 mil millones por parte del gobierno estadounidense:

El entonces secretario del Tesoro de EU, Robert Rubin, tuvo que hacer frente a esta crisis financiera. Junto con su subsecretario, Larry Summers, fueron los artífices del "paquete de rescate" que otorgó la administración Clinton por 20,000 millones de dólares, más otros 30,000 millones de "ayuda internacional" que requirió México para amortiguar la crisis que obligó a establecer un sistema de libre flotación (CNN, 2011, p. 1).

Por otra parte, el caso argentino se presentó a finales de los años 1990, cuando el país experi- mentó una fuerte salida de capital producto de la pérdida de confianza de los inversores en el manejo macroeconómico del país. Antes de la crisis, el país se había visto beneficiado por el ingreso de capitales externos debido a las condiciones de apertura y desregularización que se realizaban en la economía argentina.

No obstante, similar al caso mexicano, los ingresos de capitales en el período se canalizaron al sector bursátil y no se aplicó a la inversión en el sector real. A pesar de la confianza dada por los inversionistas, los capitales golondrina se retiraron al momento en que cambiaron las condiciones económicas generando importantes desequilibrios en la economía argentina (Fanelli, 1998).

\section{El caso de Chile}

La experiencia de Chile entre 1990 y 1997, es de especial importancia para esta investigación pues ha sido el país que mejor ha enfrentado al fenómeno de ingreso de capitales de corto plazo, en relación con México y Argentina comentados anteriormente. La forma como Chile manejó el proceso sirve para documentar el uso del encaje como medio para regular el ingreso de capitales de corto plazo, tal como se propone en Costa Rica.

Según lo exponen Le Fort y Lehmann (2003), durante la década de 1990, Chile experimentó una masiva entrada de capitales que atentó contra su estabilidad macroeconómica. Una de las razones que explicaron la afluencia de capitales durante ese período (1990-97), fue el aumento en las tasas de interés internas, producto de una política monetaria restrictiva aplicada por su Banco Central para contraer el gasto interno, con el afán de mantener una meta de inflación anual decreciente.

1. Debe indicarse que a diferencia de la situación actual en Costa Rica, la economía chilena en el período 199097, presentaba un crecimiento del ingreso nacional del $8 \%$ anual y superávits en las cuentas fiscales. 
Asimismo, señalan estos autores que durante el período 1984 a 1999 Chile mantuvo controlado el tipo de cambio a través de una banda cambiaria, y luego emigró a un régimen cambiario de flotación libre. Durante el período de fuerte entrada de capitales la operación de la banda cambiaria se caracterizó por una continua presión del tipo de cambio en el borde inferior o piso. Según Le Fort y Lehmman (2003) para:

...evitar que las alzas en las tasas de interés resultaran en mayores entradas de capitales externos, diluyendo el efecto del ajuste y generando presiones hacia la apreciación cambiaria, el Banco Central estableció, a partir de junio de 1991, un encaje a la entrada de capitales externos de carácter selectivo (p. 35).

El encaje consistió en un depósito obligatorio, en moneda extranjera y no remunerado, que debía constituirse en el Banco Central chileno en forma proporcional a ciertas entradas de capital (la tasa fue de 30\% durante casi todo el período), y que debía mantenerse durante un año. Este depósito resultaba en un encarecimiento del financiamiento externo que desincentivaba el ingreso de capitales, al menos por las vías de ingreso sujetas al mecanismo de encaje. Por su diseño el encaje afectaba fundamentalmente al endeudamiento de corto plazo (Le Fort y Lehmman, 2003).

Para el mercado financiero chileno el encaje constituyó una limitación a la integración financiera internacional pero en el contexto en que se impuso, brindó un mayor margen de operación a la política monetaria y evitó la profundización del ciclo económico que puede resultar de entradas masivas pero transitorias de capitales externos. Sin embargo, Le Fort y Lehmman (2003) señalan que una estrategia de política económica más eficaz habría sido una más amplia aplicación del encaje o la complementación de la política fiscal.

La evidencia empírica evaluada por estos autores les permitió concluir que si bien el encaje no eliminó los flujos de capital de corto plazo, si contribuyó de manera importante a disminuir la entrada de capitales en Chile, particularmente de corto plazo y modificó la composición del financiamiento externo favoreciendo flujos de mediano y largo plazo, en el decenio 1990. Posteriormente, a partir del año 1998, la afluencia masiva de capitales en Chile se redujo, regresando a niveles consistentes con su economía, lo que permitió a finales de 1999 migrar a un sistema cambiario de flotación.

Para Le Fort y Lehmman (2003), la flotación cambiaria devuelve la efectividad a la política monetaria; sin embargo, ante una intensificación de las entradas de capital, como en el caso Chileno, señalan que dejar que la moneda flote puede implicar un riesgo nada despreciable de sobreapreciación cambiaria, con efectos potencialmente muy nocivos sobre la asignación de recursos y la estabilidad macroeconómica interna y externa.

\section{Costa Rica: razones por las que ingresa el capital especulativo}

Según lo establece la teoría económica, el origen de los flujos de capital financiero de corto plazo puede originarse por causas endógenas y exógenas a las economías nacionales. En primer lugar, la afluencia de estos fondos se considera endógena si es producida por la coyuntura propia de la economía, que en cierto momento puede presentar tasas de interés por encima de las tasas internacionales. En este caso, los capitales de corto plazo ingresan por arbitraje² (Diago, 2011).

Por otra parte, si la afluencia de estos capitales se produce como resultado de la valoración del riesgo-país por parte de los inversionistas internacionales, o por la aceptación de un mayor riesgo de estos inversores en procura de diversi-

2. Por arbitraje de intereses, se entiende el proceso de mover fondos hacia monedas extranjeras en mercados externos para aprovechar los intereses de inversión más altos de inversiones en el extranjero. 
ficar su cartera de activos financieros; el origen de las entradas de capital es de tipo exógeno a la economía. Sin embargo, esta segunda circunstancia explica mejor la afluencia de capitales de mediano y largo plazo, y no necesariamente el capital especulativo (Rodríguez, 2000).

Durante el segundo semestre de 2012 la economía costarricense absorbió una cantidad significativa de los flujos externos de capital de corto plazo. Como señala León (2013) la afluencia de estos capitales obedeció, principalmente, a factores endógenos, atribuidos a la diferencia en el corto plazo de las tasas de interés en colones en relación con las tasas de interés internacionales (pp. 1-2).

A su vez, la diferencia entre las tasas de interés locales e internacionales puede tener causas externas e internas. La principal causa externa consiste en la política monetaria expansiva que han seguido los países desarrollados a partir del año 2010 como recurso para superar la crisis financiera del 2008-2010. En este sentido, los países europeos y los Estados Unidos, han buscado incentivar su gasto privado mediante tasas de interés históricamente bajas.

Según informa Vargas (2013) la Reserva Federal de Estados Unidos (FED) anunció en enero de 2013 que mantendrá su tasa de interés entre cero y $0,25 \%$, al menos hasta la segunda parte del año. Este nivel tan bajo de los tipos de interés contrasta con el nivel de tasas de nuestro país, donde la Tasa Básica Pasiva se ubicó en un promedio mensual de 6,82\% durante el año 2013 (Banco Central de Costa Rica, 2013).

Como causas internas del diferencial de tasas de interés se señalan el nivel de las tasas de interés internas, el cual es mayor significativamente que los intereses a nivel internacional, y el régimen cambiario actual denominado de bandas cambiarias. Este último por cuanto la decisión de los inversionistas de colocar fondos en un país determinado no solo depende de la diferencia de tasas de intereses, sino que también depen- de de las variaciones que se espera experimente el tipo de cambio de las monedas en el futuro inmediato (o riesgo cambiario).

De esta forma, para el caso costarricense, el capital externo especulativo ingresa atraído por los mayores rendimientos que genera una inversión en colones en el corto plazo y no se encuentra expuesto a riesgo cambiario, pues se tiene la certeza de que el tipo de cambio no variará más allá de ciertos límites, dado el sistema de bandas cambiarias vigente que establece un techo y un piso para el valor de la divisa en el país.

Por tanto, el diferencial de tasas de interés per se no es la única causa interna de los movimientos de capital especulativo. Bien podría existir una discrepancia positiva entre la tasa de interés nacional y la de otro país, que si se espera un aumento o depreciación del tipo de cambio en el país con mayor tasa de interés, el diferencial de intereses no provocaría, necesariamente, movimientos de capital especulativo.

\section{La Trinidad imposible}

En su libro de texto, Carbaugh (2009) señala que una de las consecuencias que sufre un país que permite el libre flujo de capitales es que se limita su capacidad para elegir un sistema cambiario y operar a la vez una política monetaria independiente o autónoma. El mantener abierta la cuenta de capitales hace que el país solo pueda aplicar dos de los tres objetivos de política siguientes:

a) Libre flujo de capitales (cuenta de capital abierta).

b) Tipo de cambio estable.

c) Política monetaria independiente.

Según Carbaugh (2009), los economistas utilizan el término "trinidad imposible" para referirse a esta restricción que limita la autonomía económica de los bancos centrales. 
Al considerar la "trinidad imposible" las autoridades económicas de una nación pueden enfrentar tres escenarios posibles, a saber:

1. Si se busca una movilidad perfecta de capitales para integrar el mercado de capitales interno al externo (a) y un tipo de cambio estable (b), debe renunciar a la autonomía de la política monetaria (c).

2. Si se desea mantener una política monetaria autónoma (c) y movilidad perfecta de capitales (a), se deberá dejar flotar el tipo de cambio (b).

3. Si se desea mantener un tipo de cambio estable (b) y una política monetaria independiente (c) se deberán imponer restricciones a la movilidad de capitales (a).

¿Cómo decide el país si debe sacrificar un tipo de cambio estable, una política monetaria independiente o el libre flujo de capitales? La respuesta depende tanto de la coyuntura económica que atraviese como de los intereses de los sectores económicos que podrían verse afectados por una u otra escogencia.

Los sectores exportadores y de turismo preferirán un tipo de cambio estable, para lo cual habría que renunciar a la autonomía de la política monetaria o imponer restricciones a la movilidad de capitales. En el primer caso, se afectan los intereses de la banca central, y en el segundo, los intereses de la banca comercial.

Por su parte, si el Banco Central de Costa Rica (BCCR) está interesado en mantener una política monetaria independiente que le permita llegar a cierta meta de inflación, tendrá que regular la cuenta de capitales o sacrificar la estabilidad cambiaria (migrar a un régimen de flotación del tipo de cambio). En el primer caso se afectan los intereses de la banca comercial y en el segundo los intereses de los sectores ligados a la exportación y al turismo.

Finalmente, los sectores bancarios y financieros verían con buenos ojos la desregulación y apertu- ra de la cuenta de capitales para facilitar el ingreso de fondos externos de corto, mediano y largo plazo, así como una posible flotación del tipo de cambio que les permita ampliar el negocio financiero. ${ }^{3}$ Sin embargo, dichas metas obligarían a sacrificar la independencia de la política monetaria.

\section{Implicaciones del ingreso de capitales de corto plazo sobre la economía costarricense}

Para una economía pequeña y abierta como la costarricense, el ingreso masivo de capital especulativo presiona hacia la baja el tipo de cambio, pues la ley del mercado indica que al aumentar la oferta de dólares, el precio de la divisas (tipo de cambio) debe bajar. No obstante, al existir un piso para el valor del dólar, se obliga al Banco Central a adquirir las divisas excedentes, para evitar que el tipo de cambio se traslade por debajo del valor mínimo de la banda.

Como consecuencia de la defensa del piso de la banda cambiaria, el Banco Central agotó su plan de compra de Reservas Monetarias Internacionales (RMI) de US \$1500 millones que estaba programado para dos años (2012 y 2013), según el Programa monetario del 2012-2013. De esta forma, las Reservas del Banco Central mostraban a enero de 2013 una de las mayores cifras históricas cercanas a US \$7 mil millones.

En el 2012 y lo transcurrido al mes de enero del 2013 la compra de divisas por parte del Banco Central (mayoritariamente en defensa del límite inferior de la banda cambiaria) ascendió a US \$1612,9 millones, de los cuales US \$1500,0 millones formaron parte de lo autorizado por la Junta Directiva con el segundo programa de acumulación de reservas, el ritmo de adquisición fue

3. La flotación del tipo de cambio permitiría el surgimiento de otros servicios financieros y de diversos instrumentos para la cobertura de los riesgos cambiarios, como los derivados de divisas, ventas a futuro, etc. 
FIGURA 1

\section{RESERVA MONETARIAS INTERNACIONALES EN PODER DEL BANCO CENTRAL DE COSTA RICA (BCCR)}

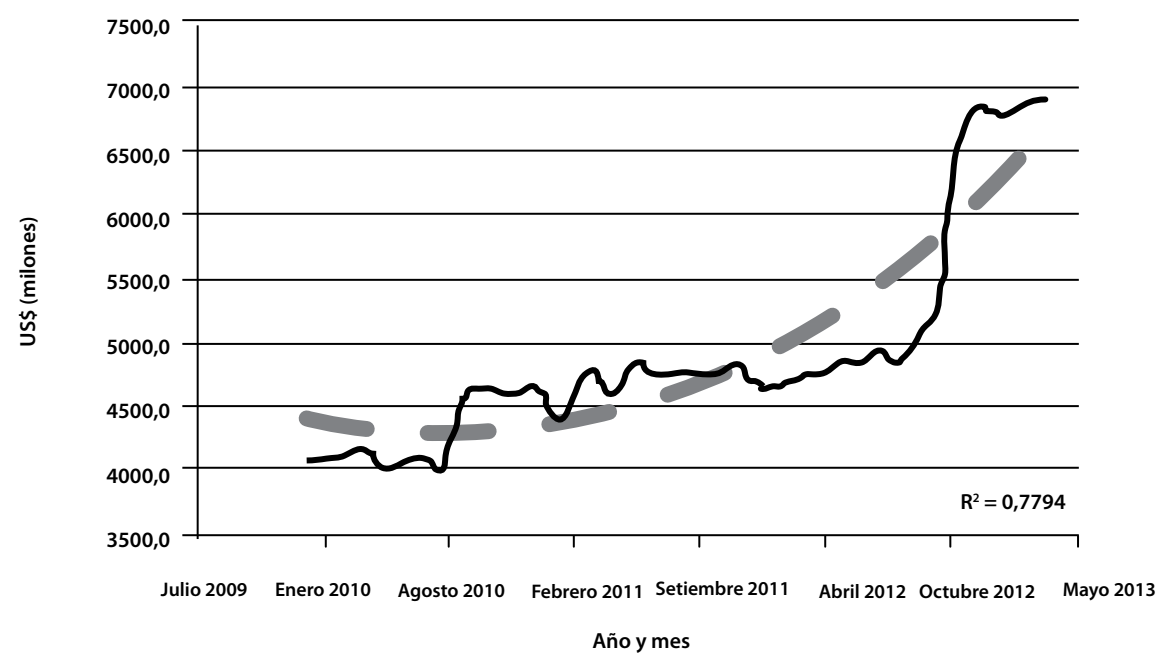

Fuente: Elaboración propia con base en datos del Banco Central de Costa Rica (2013).

mucho más rápido de lo previsto en el programa previsto para el periodo febrero 2012-diciembre 2013 (Programa Macroeconómico 2013-14).

La figura 1 muestra el crecimiento de las RMI desde enero de 2009 hasta marzo de 2013. La línea de puntos presenta la tendencia que ha mostrado la acumulación de reservas debido a la defensa del piso de la banda cambiaria. ${ }^{4}$

Para la adquisición de las divisas excedentes, el BCCR se ha visto obligado a emitir moneda local por encima de lo previsto en su programación macroeconómica, creando un exceso de demanda en los mercados de bienes y servicios por el mayor poder adquisitivo generado en el sistema económico. Esta situación presiona hacia la alza los precios internos, con lo cual se podría estar sacrificando la meta de inflación prevista por el mismo Banco:

No obstante, preocupan dos elementos, por un lado la ausencia de una solución al des-

4. El coeficiente de determinación (R2) presentado en la Figura 1, indica que el ajuste de tendencia de la acumulación de RMI modelado con una función exponencial, explica el 78\% de la variabilidad de los datos reales. equilibrio de las cuentas fiscales y, relacionado con lo anterior, el ingreso de recursos externos motivado por el arbitraje de tasas de interés, particularmente, a partir del último trimestre del 2012, que generó un desequilibrio en la cuenta financiera de la balanza de pagos, incrementó la vulnerabilidad externa del país en el corto y mediano plazo y presionó a la apreciación del colón, lo cual requirió de una mayor compra de divisas por parte del Banco Central, condición última que provocó una expansión monetaria. (Banco Central, 2013, p. 1).

Cabe indicar que un proceso inflacionario puede ser un mecanismo de ajuste per se (cuando el tipo de cambio se mantiene estable), pues al subir el nivel de precios, el colón pierde poder adquisitivo y se reduce el tipo de cambio real. Esto desmotiva el ingreso de los capitales especulativos. Sin embargo, desde el punto de vista socioeconómico el ajuste por inflación no es una política deseable, dadas las consecuencias funestas que dicho fenómeno tiene sobre los agentes económicos (hogares y empresas). 
Ahora bien, el BCCR puede buscar mantener su meta de inflación al esterilizar la emisión monetaria en exceso ${ }^{5}$, pero únicamente podrá hacerlo ofreciendo títulos en el mercado monetario, pues el artículo 63 de su Ley Orgánica le impuso un límite a la fijación de los encajes mínimos legales en 15\%.

Al ofrecer títulos en el mercado de valores, el BCCR debe proponer tasas de interés competitivas con lo cual se convierte en parte del problema, al presionar a la alza los intereses internos. Esto favorece la entrada de capitales de corto plazo, y además, incrementa las pérdidas del $B C C R$, que en última instancia se adicionan al déficit del sector público costarricense. ${ }^{6}$

Como se observa, el ingreso masivo de capitales en un contexto de tipo de cambio rígido tiene diferentes consecuencias desestabilizadoras de la economía: (a) acelera la inflación, (b) aumenta los tipos de interés, (c) aumenta las pérdidas del sector público. Todo ello tiene repercusiones negativas sobre la producción y el nivel de empleo en la economía.

\section{Políticas para reducir el ingreso de capitales especulativos}

Debido a que la atracción de capitales de corto plazo se explica por el nivel actual de la tasa de interés internos y la ausencia de riesgo cambiario se

5. La esterilización monetaria se refiere a las acciones del Banco Central para neutralizar los cambios en la oferta monetaria interna debidos a los impactos asociados a los movimientos internacionales de divisas. En este caso, si el Banco Central se ve forzado a emitir moneda para adquirir las divisas que ingresan a la economía con el fin de evitar que la oferta monetaria se amplíe, deberá recoger la moneda emitida utilizando algún otro instrumento de política monetaria, como la venta de bonos en el mercado abierto.

6. Las pérdidas del Banco Central es la diferencia entre sus ingresos (intereses, comisiones, intermediación cambiaria, entre otros) y los egresos (Intereses pagados por títulos, bonos en moneda nacional y extranjera y gastos administrativos). Estas pérdidas se suman al resultado financiero del sector público global. han propuesto dos tipos de solución: (a) reducir las tasas de interés internas y (b) eliminar las bandas cambiarias y migrar hacia un sistema de flotación del tipo de cambio (Semanario El Financiero, 2013).

La primera opción requiere una mayor investigación sobre las causas internas que mantienen las tasas de interés en el país por encima de las tasas internacionales. Si bien, se aduce que el déficit fiscal es el causante de las altas tasas de interés domésticas, aún no se ha precisado en cuánto contribuye este factor al nivel y alza de estas tasas, ni qué debemos entender en este contexto por ese déficit fiscal: ¿déficit del Gobierno Central? ¿Déficit del Sector Público Global? Y dentro del Sector Público Global, ¿cuál déficit es el relevante? ¿Déficit del Sector Público No Financiero (SPNF) o el déficit del BCCR?

En el Programa Macroeconómico 2013-14, el Banco Central estima que para 2013 las finanzas del Gobierno Central cerrarán con un déficit de 4,8\% del PIB (5,3\% para el Sector Público Global) y para 2014 de 5,0\% (5,4\% para el Global). El BCCR prevé que este déficit continuará financiándose con colocación de bonos de deuda interna. No obstante, es preciso señalar que el resultado financiero del Sector Público Global se compone, para 2013, de un déficit de 4,8\% del PIB del Gobierno Central, un superávit de 0,3\% del resto del Sector Público No Financiero (SPNF) y un déficit de 0,9\% del BCCR.

Debido a lo anterior, y aun desconociendo el nivel efectivo de presión que ejerce esa variable sobre las tasas de interés, al parecer el mayor problema se encuentra en el déficit del Gobierno Central, pues se estima que el resto del Sector Público No Financiero presenta un superávit.

Otra de las causas del elevado nivel de las tasas de interés internas se puede hallar en las medidas de política monetaria que debe aplicar el BCCR para mantener la meta de inflación planteada en su Programa Macroeconómico 2013-2014. Debido al ingreso de capitales externos que vienen a ser in- 
vertidos en títulos valores en el mercado financiero costarricense, el BCCR ha debido adquirir estas divisas para evitar una mayor apreciación del tipo de cambio (defensa del piso de la banda cambiaria). Con este accionar, el BCCR aumenta la liquidez en la economía con lo cual presiona al alza el nivel de precios y compromete el objetivo de inflación indicado en el Programa Macroeconómico.

De esta forma, en aras de mantener la meta inflacionaria y no socavar la confianza del público en la política monetaria, el BCCR debe eliminar (esterilizar) la liquidez creada por la compra de moneda extranjera, y para ello puede recurrir a colocar títulos o bonos en el mercado abierto. Esta otra medida limita la baja en las tasas de interés internas, con lo cual se mantiene la causa interna que estimula el ingreso de capitales.

Para controlar la liquidez en la economía, además de las operaciones de mercado abierto, el BCCR posee otro instrumento de control monetario que no aumenta las pérdidas del ente emisor. Este instrumento es el encaje mínimo legal. Pero la utilización de esta herramienta se encuentra limitada por la Ley Orgánica del Banco Central de Costa Rica, que impone un límite del 15\% para el encaje mínimo legal. ${ }^{7}$

Por otra parte, la segunda opción, migrar hacia un sistema de flotación cambiaria administrada, eliminaría hasta cierto punto la certeza o previsibilidad cambiaria que poseen los agentes económicos en cuanto al comportamiento del tipo de cambio, introduciendo incertidumbre por el riesgo cambiario en los inversores extranjeros, lo cual contribuye a limitar el ingreso indiscriminado de capitales especulativos. Sin embargo, producto de este mismo proceso que ocasiona el ingreso del capital especulativo durante estos

7. Una forma de brindar una mayor flexibilidad a la política monetaria sería eliminar estas restricciones al encaje establecidas en el marco normativo de Banco Central, para que este instrumento retome su importancia como medio de control monetario. dos años, el tipo de cambio ha tendido a la baja, ubicándose en el piso de la banda (\$500) y no ha disminuido más por la intervención del BCCR, como se indicó anteriormente.

Por lo tanto, el temor en adoptar esta opción (flotación del tipo de cambio), descansa en la creencia de que si al eliminar la banda, el tipo de cambio bajaría aún más favoreciendo la entrada de capitales especulativos y la importación. Esto afecta de forma negativa al sector exportador y al sector turístico y demás actividades encadenadas con estos sectores.

A pesar de lo anterior, también se reconoce que de cumplirse este pronóstico, habría sectores beneficiados, como señala el economista Luis Paulino Mora (2013): "En contrapartida se lograría reducir la inflación y abaratar los productos importados, en beneficio del "consumidor" y con gran contento para el comercio importador. Usualmente se omite decir que también beneficiaría a la banca, la cual podrá así inaugurarse en el casino de la especulación cambiaria".

Quizás una opción que debe valorarse y que ha sido poco discutida es la baja del piso de la banda, de manera que se alivie la presión del BCCR en absorber los excedentes de dólares del mercado cambiario y pueda tener un mayor margen de maniobra para lograr la meta de inflación establecida en su programación monetaria. Además, si el tipo de cambio continua bajando se favorece aún más la entrada de capitales especulativos.

\section{El proyecto "Ley para desincentivar el ingreso de capitales externos"}

Durante el segundo semestre del año 2013 se discutió en la corriente legislativa el Proyecto N. 18685 denominado: Ley para desincentivar el ingreso de capitales externos. En la presentación de este Proyecto, el Poder Ejecutivo aduce al ingreso de capitales de corto plazo producto de la coyuntura económica del año 2012 y se señala específicamente que: 
A partir del segundo semestre del 2012 y lo transcurrido del 2013, la economía costarricense ha presentado condiciones que incentivan el ingreso de capital externo motivado por rendimientos financieros. La libre movilidad de capitales; tasas de interés en los mercados internacionales en niveles históricamente bajos; elevados rendimientos de títulos valores locales, producto de la competencia por fondos prestables por parte del sector público y el privado; y un tipo de cambio prácticamente fijo ("anclado" en el límite inferior de la banda cambiaria) aumentaron de manera significativa el premio por ahorrar en instrumentos de deuda costarricense denominados en colones. (Proyecto No 18.685, 2013, p. 1)

Básicamente, el proyecto lo que hace es adicionar un artículo 59bis a la Ley del Impuesto sobre la Renta, Ley No. 7092 del 21 de abril de 1988, y un artículo 80Bis a la Ley Orgánica del Banco Central de Costa Rica, Ley №. 7558 del 3 de noviembre de 1995.

En la propuesta de reforma a la Ley del Impuesto sobre la Renta, se autoriza al Poder Ejecutivo, por solicitud del BCCR, a incrementar hasta por un plazo de seis meses prorrogables la tarifa de los impuestos aplicables sobre intereses pagados o acreditados y descuentos concedidos a personas no domiciliadas en el país. El incremento en las tarifas podrá alcanzar hasta 30 puntos porcentuales adicionales.

Por su parte, la propuesta de reforma a la Ley Orgánica del Banco Central de Costa Rica, establece que cuando a juicio de la Junta Directiva del BCCR, se presenta un desequilibrio originado por el ingreso de capitales del exterior destinados a inversiones de corto plazo, se obliga a las entidades autorizadas para realizar transacciones en el mercado de valores costarricense, a constituir un depósito obligatorio en el BCCR no remunerado por un monto equivalente de hasta 25 por ciento de esos fondos provenientes de sus clientes no domiciliados en el país. La medida podrá ser utilizada de forma inmediata y tantas veces como sea necesaria, a partir de la terminación de su plazo de vigencia.
Si bien, el proyecto constituye un paso importante para limitar el ingreso de los capitales especulativos al país, no se considera como suficiente para resolver el problema de fondo o estructural interno que origina la entrada de estos capitales. Mientras los factores internos que ocasionan la afluencia de capitales de corto plazo persistan, al Banco Central le resultará difícil cumplir con la meta de inflación propuesta y dar el paso de un régimen de bandas cambiarias a la flotación del tipo de cambio, como originalmente se había planteado. Como se ha indicado, para mantener estable el tipo de cambio y brindar mayor margen de flexibilidad e independencia a la política monetaria se deberá fortalecer la intervención del Estado en el funcionamiento del mercado de capitales.

Debido a lo anterior, se considera que las medidas que se pretendan tomar para limitar el ingreso de estos capitales deberían enfatizar en una solución para el problema estructural, es decir, corregir las causas internas que motivan la atracción de capital especulativo (diferencial entre tasas de interés y ausencia de riesgo cambiario), más que contrarrestar los síntomas o resultados del problema (ingreso de capital especulativo), el cual es un problema de tipo coyuntural.

En este caso, las medidas propuestas en el expediente legislativo n. ${ }^{\circ} 18.685$, lo que buscan es reducir de forma coyuntural y selectiva la rentabilidad doméstica de las inversiones de capitales externos para desestimular su ingreso en situaciones específicas. Ello para mantener un tipo de cambio estable y ampliar el margen de maniobra de la política monetaria (se elige uno de los casos de la trinidad imposible).

\section{CONCLUSIONES Y RECOMENDACIONES}

La afluencia de capitales especulativos a Costa Rica a partir del segundo semestre de 2012, se explica principalmente por el diferencial entre las tasas de interés internas y externas de corto 
plazo, lo cual ha incentivado la inversión financiera en colones en el mercado interno.

Como aspecto coadyuvante, que favorece el ingreso de capitales de corto plazo, se presenta la política cambiaria vigente, que sitúa al tipo de cambio dentro de una banda de precios con una clara tendencia a mantenerse cercano al piso o valor mínimo de la banda; además de una recurrente intervención del Banco Central comprando moneda extranjera para evitar que el tipo de cambio se reduzca por debajo de ese piso.

Con la finalidad de controlar o regular las entradas de capital golondrina se analiza en el Poder Legislativo un proyecto de Ley que propone dos instrumentos discrecionales. Como se indicó anteriormente, el primero es de tipo tributario y consiste en un incremento transitorio del impuesto sobre la renta para estos capitales. El segundo instrumento consiste en el establecimiento de un encaje legal adicional que inmovilizaría el 25\% de los depósitos de capital sin contemplar remuneración alguna.

El hecho de que las autoridades económicas hayan apostado por incrementar la regulación de los flujos de capital de corto plazo es un claro indicio de su posición ante "La trinidad imposible". En este caso, las autoridades están eligiendo como objetivos mantener un tipo de cambio estable y preservar la independencia de política monetaria, por tanto, se sacrifica el objetivo de mantener un libre flujo de capitales, es decir, se abandona el objetivo de mantener abierta la cuenta de capital de la balanza de pagos.

Debido a lo anterior, se procederá a revisar y comentar cada uno de los instrumentos de regulación propuestos a la Asamblea Legislativa.

El primer instrumento es la creación de un nuevo artículo 59bis en la Ley del Impuesto sobre la Renta, mediante el cual el Estado tendrá la facultad de aumentar, de forma transitoria y hasta por seis meses, las tarifas impositivas sobre los inte- reses que se paguen a agentes no domiciliados por los emisores, agentes pagadores y otras sociedades públicas o privadas del país, originados en la entrada de capitales extranjeros.

El nuevo impuesto que se estaría creando lo que pretende es restringir transitoria y no permanentemente las entradas de capitales de corto plazo, cuando a juicio de la Junta Directiva del BCCR, se considere que su arribo atenta contra la estabilidad o equilibrio de la economía. La aplicación del impuesto por parte del Poder Ejecutivo quedaría sometida a un acuerdo de mayoría calificada por parte de la Junta Directiva del BCCR.

Según el artículo 59bis propuesto, se establece que ante el acuerdo del Banco Central, el Poder Ejecutivo queda obligado a incrementar las tarifas de los impuestos al capital externo. No obstante, en el segundo párrafo se indica que el acuerdo de la Junta Directiva del Banco Central lo que viene es a recomendar los incrementos y plazos en las tarifas tributarias y que el Ejecutivo podrá establecer incrementos y plazos diferenciados a las tarifas de retención entre distintas monedas.

Se observa que el texto de la propuesta no es coherente en cuanto al mecanismo de coordinación o de subordinación entre ambas entidades (Poder Ejecutivo y Banco Central), para la aplicación de la medida impositiva, considerando que si la aplicación de las disposiciones dadas en el acuerdo del BCCR son de acatamiento obligatorio para el Poder Ejecutivo, hasta cierto punto se estará facultando a la Autoridad Monetaria a tomar decisiones en el ámbito fiscal, el cual es propio del Poder Ejecutivo (se vulnera la independencia entre el ente monetario y el ente fiscal). En este sentido, si se pretende subordinar un poder sobre otro es recomendable que el ente proponente reflexione sobre la conveniencia de esta circunstancia.

Por otra parte, el mecanismo o procedimiento de activación del nuevo impuesto se considera que debería quedar establecido en la Ley que se 
emita, pues el procedimiento; tal como se establece en el proyecto de ley en este momento, pueda trasgredir el principio de reserva de ley en materia tributaria. En este sentido, es conveniente la revisión de un procedimiento similar al planteado en la propuesta de ley y que se aplica en la Ley de Consolidación de Impuestos Selectivos de Consumo, Ley n. 4961 y sus reformas.

En la Ley de Impuestos selectivos, se faculta al Poder Ejecutivo a la aplicación por excepción de una tarifa tributaria adicional en casos de "extrema urgencia fiscal". Específicamente, en el artículo 11 se indica de forma explícita las mercancías a las que se aplica el impuesto selectivo y en el artículo 12, denominado flexibilidad, se define qué debe entenderse por "situaciones de extrema urgencia fiscal", así como el mecanismo de coordinación con la Asamblea Legislativa para salvaguardar el principio de reserva de ley. La aplicación de impuestos selectivos de consumo, las situaciones de "extrema urgencia fiscal" se definen en el artículo 12 de la siguiente manera:

Para los efectos de la validez de este aumento temporal, se denomina "extrema urgencia fiscal" la disminución proyectada al cierre del ejercicio económico de más de un cinco por ciento (5\%) de los ingresos probables estimados en el presupuesto de la República vigente en el momento de la emisión del decreto ejecutivo correspondiente. La metodología para demostrar la proyección de disminución de ingresos, será definida en el Reglamento de esta Ley, como requisito de validez de la discrecionalidad tarifaria aquí contemplada (Ley n. ${ }^{\circ} 4961$, artículo 12)

Asimismo, el procedimiento de coordinación entre el Poder Ejecutivo y el Poder Legislativo para proteger el principio de reserva de ley se establece de la manera siguiente:

El incremento en las tarifas será aplicable por un plazo máximo de seis meses y requerirá la ratificación, por parte de la Asamblea
Legislativa, del decreto ejecutivo en que se establece el aumento. La Asamblea dispondrá de un plazo de un mes para pronunciarse sobre el respectivo decreto en una sola sesión plenaria, plazo que empezará a contar desde que ese decreto sea leído por el Directorio de la Asamblea Legislativa. El Directorio tendrá un plazo de seis días hábiles desde la recepción del decreto para leerlo, como requisito de validez. El silencio de la Asamblea Legislativa sobre el decreto en el término indicado, deberá entenderse como consentimiento. Dicho decreto entrará en vigencia a partir del primer día hábil del mes siguiente a la fecha de la aprobación, expresa o tácita, de la Asamblea Legislativa. Salvo lo indicado en los párrafos anteriores, para restituir o aumentar las tarifas referidas en el Anexo a esta Ley, se requerirá la aprobación legislativa (Ley n. ${ }^{\circ}$ 4961, artículo 12)

Ninguno de los elementos anteriores se observan en la propuesta del nuevo artículo 59bis que se adicionaría a la Ley del Impuesto sobre la Renta, ni procedimientos similares, acordes con la aplicación del nuevo tributo, deberían valorarse en la propuesta sometida a la Asamblea Legislativa.

En la misma línea del espíritu de la reforma planteada, se considera oportuno analizar la posibilidad de reformar propiamente el artículo 59 de la Ley del Impuesto sobre la Renta, Ley n. 7058 , para someter al pago de impuestos a los intereses, comisiones y otros gastos financieros pagados por empresas domiciliadas en el país a entidades financieras del exterior. Ello porque en la redacción actual de ese artículo, se exonera del pago de impuesto sobre la renta a estos capitales, en el tanto las entidades financieras que los gestionan sean reconocidas por el BCCR como bancos de primer orden o entidades dedicadas a operaciones internacionales.

Esta situación no permite aumentar la recaudación fiscal en renta lo cual no favorece la re- 
ducción del déficit del Gobierno Central y a la vez estimula el ingreso de capitales externos de corto plazo, con las consecuencias ya conocidas sobre la estabilidad macroeconómica y que, precisamente, el proyecto de Ley propuesto por el Ejecutivo busca solventar. Esta reforma, sería una medida menos distorsionante para la aplicación del impuesto sobre la renta, que la sugerida con la inclusión del nuevo artículo 59bis propuesto.

El segundo instrumento propuesto en el Proyecto de Ley para desincentivar el ingreso de capitales es la adición de un nuevo artículo 80bis a la Ley Orgánica del Banco Central de Costa Rica, Ley n. 7558 y sus reformas. Este nuevo artículo faculta al Banco Central a exigir un depósito obligatorio no remunerado de hasta $25 \%$ sobre los capitales externos destinados a inversiones en títulos del mercado de valores costarricense. Este depósito previo es similar a un encaje específico, a nuestro criterio, muy similar al caso chileno comentado anteriormente.

De la experiencia chilena en el periodo 1990-97 reseñada anteriormente, la aplicación de un encaje de $30 \%$ a las entradas de capital de corto plazo fue catalogada como una política exitosa para controlar el ingreso de dichos capitales. Empero, los analistas de esta política indicaron que debió utilizarse de forma más amplia

A causa de lo anterior, una recomendación es valorar la apertura de este instrumento de manera que a juicio del BCCR (por votación calificada), pueda ser aplicado a los ingresos de capital externo en general y no como está en la actual propuesta que lo restringe, únicamente, al ingreso de capitales del exterior destinados a inversiones en títulos inscritos en el Registro Nacional de Valores e Intermediarios.

Asimismo, se considera recomendable que la Asamblea Legislativa valore la conveniencia de que en el proyecto de Ley se fije un límite a estos depósitos externos (según el proyecto el límite sería de 25\%). Debido a ello, se considera que el porcentaje de los depósitos previos debería ser determinado técnicamente por el BCCR (en su acuerdo por votación calificada), según la magnitud de los ingresos de capital, el desequilibrio que se necesita corregir y las condiciones macroeconómicas del momento.

Adicionalmente, es oportuno que dentro de esta propuesta de ley, se reforme o se derogue el artículo 63 de la Ley Orgánica del Banco Central de Costa Rica, sobre el límite del encaje mínimo legal, con la finalidad de eliminar el límite máximo de 15\%, el cual actualmente constituye una restricción para el uso de este instrumento de política monetaria y que obliga al ente emisor a recurrir a mecanismos más costosos para la economía cuando requiere retirar liquidez del sistema.

Este límite en el uso del encaje mínimo legal ha forzado al BCCR a controlar la liquidez del mercado financiero a través de operaciones de mercado abierto, con la consecuente presión sobre las tasas de interés domésticas y el incremento en las pérdidas del BCCR que engrosan el déficit del sector público global. Asimismo, se considera fundamental que se posibilite al BCCR a aplicar la política de encaje a todas las entradas de capitales internacionales, de acuerdo con el criterio y juicio de su Junta Directiva.

Finalmente, es necesario dejar patente que el proyecto de ley lo que busca es regular el ingreso de los capitales externos de corto plazo y no constituye una medida para corregir las causas internas que originan dicho proceso. Por esta razón, se considera oportuno que, en complemento al proyecto de ley en cuestión, se legisle en procura de una solución al problema estructural que origina el elevado nivel de las tasas de interés y que, en última instancia, incentiva la llegada de capitales especulativos.

De acuerdo con la opinión de diversos especialistas, la presión alcista en las tasas de interés se produce por las necesidades de financiamiento 
del déficit fiscal del Gobierno Central. En ese sentido, una reforma fiscal enmarcada en los principios de solidaridad, eficiencia y equidad, que venga a corregir la regresividad de la estructura actual y la evasión, se hace indispensable.

Finalmente debe indicarse que la discusión legislativa del proyecto de ley para frenar el ingreso de capitales de corto plazo, a pesar de contar con los votos para su aprobación, se trasladó para el año 2014, debido al receso legislativo aprobado por el período electoral que finaliza en el primer cuatrimestre de 2014.

\section{REFERENCIAS}

Asamblea Legislativa del gobierno de Costa Rica. Departamento de Servicios Parlamentarios. Proyecto de Ley n. ${ }^{\circ} 18.685$ (2013). Ley para desincentivar el ingreso de capitales externos. Recuperado de http://www.asamblea. go.cr/Centro_de_informacion/Consultas_SIL/Pginas/ Proyectos\%20de\%20ley.aspx

Banco Central de Costa Rica. (2013). Programa Macroeconómico 2012-13. Recuperado de http://www.bccr.fi.cr/

Banco Central de Costa Rica. (2013). Programa Macroeconómico 2013-14. Recuperado de http://www.bccr.fi.cr/

Banco Central de Costa Rica. (2013). Tasa básica mensual. Recuperado de: http://indicadoreseconomicos.bccr. fi.cr/indicadoreseconomicos/Cuadros/frmVerCatCuadro.aspx?idioma=1\&CodCuadro=\%20592

Carbaugh, R. (2009). Economía Internacional (12a. Ed.). México: Cengage Learning.

CNN. (2011). El efecto tequila. Recuperado de http://www.cnnexpansion.com/economia/2011/07/28/el-efecto-tequila

Diago, F. (2011). Capitales golondrina, su incidencia en países emergentes: caso Colombia. Revista Dictamen Libre, 8, pp.53-58.

El Financiero (2013). Medidas para controlar ingreso de capitales podrían quedarse cortas: Margen de acción del Poder Ejecutivo en reducción de tasa de interés es limitado. Recuperado de: http://www.elfinancierocr.com/ finanzas/Medidas-controlar-capitales-podrian-quedarse_0_228577148.html

Fanelli, J. (1998). Regulación de los movimientos de capital en los países en desarrollo: reflexiones sobre la experiencia de los noventa. Banco Central de Bolivia. Recuperado de http://www.bcb.gob.bo/webdocs/2011/Publicaciones/RevistaAnalisis/1998/dic/cap6\%20final.pdf

Gastaldi, S y Ferro, G. (1995). La crisis mexicana de diciembre de 1994 y las dificultades de la convertibilidad y del sistema financiero argentino. Recuperado de http:// www.fundacionege.org/publicaciones/miembros/ libros/1995/03/31/efecto_tequila.pdf

Hernández, R., Fernández, C. \& Baptista, P. (2003). Metodología de la investigación. México: Mc Graw Hill.

Le Fort, G. y Lehmann, S. (2003). El encaje y la entrada neta de capitales: Chile en el decenio de 1990: Revista de la CEPAL, 81, pp. 33-64.

León, J. (2013). Capital Inflows in a Small Open Economy: Costa Rica. WorkDocument DT-03-2013. Banco Central de Costa Rica. pp. 1-13. Recuperado de http://mpra. ub.uni-muenchen.de/44512/

Lopera, C., Rojas, L. \& Sánchez, D. (2008). Capital Golondrina. Recuperado de http://carlosmario626.blogspot. com/2008/07/trabajo-final-capitales-golondrina.html

Ministerio de Hacienda de Costa Rica. (2013). Impuesto selectivo de consumo. Recuperado de http://dgt.hacienda.go.cr/tiposdeimpuestos/Paginas/ImpuestoSelectivodeConsumo.aspx

Ministerio de Hacienda de Costa Rica. (2013). Ley 7092: Ley del/mpuesto sobrela renta. Recuperado de http://www. hacienda.go.cr/Msib21/Espanol/Direccion+Juridica/ Cevcoh/Documentos_Cevcoh_5/MI_8f591aa92f2b44 7c818370e42f592ff5.htm

Mora, L. (2013). ¿Liberalizamos el dólar? Recuperado de http://sonarconlospiesenlatierra.blogspot.com/

Rodríguez, M. (2000). Crisis Monetarias y Racionalidad de Ios AtaquesEspeculativos: Una Aplicación del Modelo de Markov con Saltos de Régimen. Tesis doctoral para el grado de doctora en ciencias económicas y empresariales. Universidad de Valladolid, España.

Samuelson, P. y Nordhaus, W. (2010). Macroeconomía: con aplicaciones a Latinoamérica (19a Ed.). México: Mc Graw Hill.

Vargas, M. (2013). La FED Mantendrá tasas de interés en 0,25\%. Grupo Financiero Acobo. Recuperado de http:// www.grupoacobo.com/fed-mantendra-las-tasas-deinteres-en-0-25/

Recibido: 28 de mayo de 2013 Aceptado: 11 de noviembre de 2013 